\title{
Asymptotic Behavior of the Emptiness Formation Probability in the Critical Phase of XXZ Spin Chain
}

\author{
V. E. Korepin \\ C.N. Yang Institute for Theoretical Physics \\ State University of New York at Stony Brook \\ Stony Brook, NY 11794-3840, USA \\ S. Lukyanov \\ Department of Physics and Astronomy, Rutgers University \\ Piscataway, NJ 08855-0849, USA \\ Y. Nishiyama \\ Department of Physics, Faculty of Science, Okayama University \\ Okayama 700-8530, Japan \\ M. Shiroishi \\ Institute for Solid State Physics, University of Tokyo \\ Kashiwanoha 5-1-5, Kashiwa, Chiba, 277-8571, Japan
}

October 26, 2018

\begin{abstract}
We study the Emptiness Formation Probability (EFP) for the spin 1/2 $X X Z$ spin chain. EFP $P(n)$ detects a formation of ferromagnetic string of the length $n$ in the ground state. It is expected that EFP decays in a Gaussian way for large strings $P(n) \sim n^{-\gamma} C^{-n^{2}}$. Here, we propose the explicit expressions for the rate of Gaussian decay $C$ as well as for the exponent $\gamma$. In order to confirm the validity of our formulas, we employed an $a b$ initio simulation technique of the density-matrix renormalization group to simulate $X X Z$ spin chain of sufficient length. Furthermore, we performed Monte-Carlo integration of the Jimbo-Miwa multiple integral for $P(n)$. Those numerical results for $P(n)$ support our formulas fairly definitely.
\end{abstract}


We consider interacting spin $1 / 2$ on the one-dimensional infinite lattice. The model is described by the Hamiltonian

$$
\mathcal{H}=\sum_{j=-\infty}^{\infty}\left\{S_{j}^{x} S_{j+1}^{x}+S_{j}^{y} S_{j+1}^{y}+\Delta\left(S_{j}^{z} S_{j+1}^{z}-\frac{1}{4}\right)\right\},
$$

where $S=\sigma / 2$ and $\sigma$ are Pauli matrices. It exhibits diverse physics with varying the anisotropy parameter $\Delta$. For $\Delta \leq-1$ the ground state has a long-range ferromagnetic order, and a finite excitation gap opens in the low-lying spectrum. For $\Delta>1$ the ground state develops antiferromagnetic order, the gap also exists. For moderate anisotropy $-1<\Delta \leq 1$, the antiferromagnetic order is dissolved by quantum fluctuations. The gap closes, and the long-range asymptotic of correlation function $\left\langle S_{i} S_{j}\right\rangle$ decays as a power law at zero temperature.

In this article we study the Emptiness Formation Probability (EFP),

$$
P(n)=\left\langle\prod_{j=1}^{n}\left(S_{j}^{z}+\frac{1}{2}\right)\right\rangle,
$$

in the domain of critical phase. The importance of EFP was emphasized in the book [6]. It reflects the nature of the ground-state and it is a good indicator of the phase-separation.

In spite of its seemingly complicated definition, EFP has emerged quite naturally in the course of studies of the quantum integrability of the $X X Z$ spin chain. The first progress in calculating of EFP was achieved in Ref. [1], where $P(3)$ was obtained for the isotropic antiferromagnetic chain $(\Delta=1)$. Subsequently, Jimbo and Miwa derived multiple integral representation for all correlation functions by means of vertex operators approach [2, 3]. Their integral formula for EFP is:

$$
\begin{aligned}
P(n)=(-\nu)^{-} & \frac{n(n+1)}{2} \int_{-\infty}^{\infty} \frac{\mathrm{d} \lambda_{1}}{2 \pi} \cdots \int_{-\infty}^{\infty} \frac{\mathrm{d} \lambda_{n}}{2 \pi} \prod_{a>b} \frac{\sinh \left(\lambda_{a}-\lambda_{b}\right)}{\sinh \left(\left(\lambda_{a}-\lambda_{b}-\mathrm{i} \pi\right) \nu\right)} \\
& \times \prod_{k=1}^{n} \frac{\sinh ^{n-k}\left(\left(\lambda_{k}+\mathrm{i} \pi / 2\right) \nu\right) \sinh ^{k-1}\left(\left(\lambda_{k}-\mathrm{i} \pi / 2\right) \nu\right)}{\cosh ^{n}\left(\lambda_{k}\right)}
\end{aligned}
$$

where the parameter $\nu$ is related to the anisotropy $\Delta$ as

$$
\nu=\frac{1}{\pi} \cos ^{-1}(\Delta)
$$

The integral representation was reproduced in the framework of algebraic Bethe ansatz in 酒. A good deal of new developments have been reported for EFP. For example, in the case of $\Delta=1 / 2$ the simple formula for $P(n)$ has been conjectured in [10] and then proved in [11]. For $\Delta=0$ the asymptotic form of $P(n)$ as $n \gg 1$ was found in [5]. In either case the large- $n$ asymptotic behavior is given by $P(n) \sim n^{-\gamma} C^{-n^{2}}$ with

$$
\begin{array}{lll}
C=\sqrt{2}, & \gamma=\frac{1}{4}, & (\nu=1 / 2), \\
C=\frac{8}{3 \sqrt{3}}, & \gamma=\frac{5}{36}, & (\nu=1 / 3) .
\end{array}
$$

Moreover, $P(n)$ was calculated recently for $\Delta=1$ for strings of the length $n \leq 6$, see papers [7, 8, 9]. Their result suggests that $P(n)$ at $\Delta=1$ decays in a Gaussian way as well. Meanwhile, the asymptotic behavior of EFP for the whole critical regime was 
analyzed in field-theoretical framework, by Abanov and Korepin [12]. The Gaussian decay naturally appears in this approach.

Based on those developments, it would appear reasonable that the following asymptotic form of EFP

$$
P(n) \simeq A n^{-\gamma} C^{-n^{2}},
$$

holds for all over the critical regime $(-1<\Delta \leq 1)$. We propose the explicit expressions for the rate of Gaussian decay $C$ and the power-law exponent $\gamma$ :

$$
\begin{aligned}
C & =\frac{\Gamma^{2}(1 / 4)}{\pi \sqrt{2 \pi}} \exp \left\{-\int_{0}^{\infty} \frac{\mathrm{d} t}{t} \frac{\sinh ^{2}(t \nu) \mathrm{e}^{-t}}{\cosh (2 t \nu) \sinh (t)}\right\} \\
\gamma & =\frac{1}{12}+\frac{\nu^{2}}{3(1-\nu)}
\end{aligned}
$$

One can confirm that above formulas reproduce the exact results for $\Delta=0$ and $\Delta=1 / 2$ (5).

In order to confirm the validity of the formulas for general anisotropies $0 \leq \nu<1$, we have performed extensive numerical calculations of two kinds: One is the first-principle simulation method, namely, the density-matrix renormalization group (DMRG) 13, 14, 15], and the other is the Monte-Carlo numerical integration of the multiple integration formula (3) of Jimbo and Miwa. As noted afterwards, those methods are compensative, and we were able to perform reliable simulations for over all the critical regime. As a result, we could confirm fairly definitely that the above general formulas are indeed correct.

Let us turn to addressing the numerical-simulation results. In Tables 1-4, we have listed the DMRG results of EFP for $\nu=0.2, \ldots, 0.8$. (The methodological details will be explained afterwards.)

In addition, in each table, we have presented the logarithm of the ratio of two adjacent EFPs, which should behave in the form

$$
\ln \left(\frac{P(n)}{P(n+1)}\right) \simeq \gamma \ln \left(1+\frac{1}{n}\right)+(2 n+1) \ln C,
$$

according to the long-distance asymptotic formula (6). Note that, after taking the ratio, we are able to kill the contribution of the constant factor $A$ of (6), for which, at present, we have no analytical prediction. (We, however, could estimate $A$ numerically from our DMRG data as is shown in Table 5.) In this way, the resultant processed data can be directly comparable with the analytical conjecture.

We see that our processed DMRG data $\ln (P(n) / P(n+1))$ are extremely close to the analytical prediction (9). Actually we find that they coincide up to two or three digits for general values of $n$ and $\nu$. Therefore we conclude that our formulas for the asymptotic form are valid indeed for all over the regime $0 \leq \nu<1$. Especially see the Table 6 , where we compare the asymptotic formula with the known exact values for $\Delta=1[7,8,9]$. Note that, in this case, we have $C=\Gamma^{2}(1 / 4) /(\pi \sqrt{2 \pi})=1.66925 \ldots, \quad \gamma=1 / 12$, and $A=0.841$.

We, however, remark that rather large discrepancies are seen for such cases either very large $n$ for small $\nu$ or small $n$ for large $\nu$. The former deviations are merely due to the numerical round off errors. Since in computers, real numbers are stored in 8-byte space, and the precision is of the order of $10^{-15}$ at best. Hence it is in principle difficult to calculate the correlations less than the magnitude $<10^{-12}$ reliably. The latter deviations are not so surprising, because our general formulas should be justified for long distances of EFP. This short-range deviation will be further exploited in the succeeding Monte-Carlo numerical integration analyses.

So far, we had analyzed processed EFP data. In Fig. 1, we present the raw (unprocessed) EFP data obtained with the DMRG method. The dotted lines are the analytical 
Table 1: DMRG data for $P(n): \nu=0.2$

\begin{tabular}{cccc}
\hline$n$ & $P(n)$ & $\ln (P(n) / P(n+1))$ & Asymptotics (9) \\
\hline 1 & 0.5 & 1.505 & 1.512 \\
2 & $1.111 \times 10^{-1}$ & 2.447 & 2.447 \\
3 & $9.614 \times 10^{-3}$ & 3.397 & 3.397 \\
4 & $3.218 \times 10^{-4}$ & 4.353 & 4.353 \\
5 & $4.140 \times 10^{-6}$ & 5.312 & 5.312 \\
6 & $2.041 \times 10^{-8}$ & 6.276 & 6.271 \\
7 & $3.837 \times 10^{-11}$ & 7.187 & 7.232 \\
8 & $2.901 \times 10^{-14}$ & 4.683 & 8.192 \\
9 & $2.682 \times 10^{-16}$ & 3.923 & 9.155 \\
\hline
\end{tabular}

Table 2: DMRG data for $P(n): \nu=0.4$

\begin{tabular}{cccc}
\hline$n$ & $P(n)$ & $\ln (P(n) / P(n+1))$ & Asymptotics (9) \\
\hline 1 & 0.5 & 1.319 & 1.320 \\
2 & $1.337 \times 10^{-1}$ & 2.070 & 2.071 \\
3 & $1.687 \times 10^{-2}$ & 2.851 & 2.851 \\
4 & $9.752 \times 10^{-4}$ & 3.640 & 3.640 \\
5 & $2.561 \times 10^{-5}$ & 4.434 & 4.433 \\
6 & $3.039 \times 10^{-7}$ & 5.231 & 5.230 \\
7 & $1.626 \times 10^{-9}$ & 6.026 & 6.025 \\
8 & $3.924 \times 10^{-12}$ & 5.862 & 6.823 \\
9 & $1.117 \times 10^{-14}$ & 2.824 & 7.621 \\
\hline
\end{tabular}

Table 3: DMRG data for $P(n): \nu=0.6$

\begin{tabular}{cccc}
\hline$n$ & $P(n)$ & $\ln (P(n) / P(n+1))$ & Asymptotics (9) \\
\hline 1 & 0.5 & 1.106 & 1.125 \\
2 & $1.655 \times 10^{-1}$ & 1.594 & 1.588 \\
3 & $3.360 \times 10^{-2}$ & 2.121 & 2.115 \\
4 & $4.028 \times 10^{-3}$ & 2.667 & 2.663 \\
5 & $2.798 \times 10^{-4}$ & 3.223 & 3.221 \\
6 & $1.115 \times 10^{-5}$ & 3.784 & 3.783 \\
7 & $2.534 \times 10^{-7}$ & 4.348 & 4.348 \\
8 & $3.278 \times 10^{-9}$ & 4.909 & 4.915 \\
9 & $2.420 \times 10^{-11}$ & 5.231 & 5.483 \\
\hline
\end{tabular}


Table 4: DMRG data for $P(n): \nu=0.8$

\begin{tabular}{cccc}
\hline$n$ & $P(n)$ & $\ln (P(n) / P(n+1))$ & Asymptotics $(9)$ \\
\hline 1 & 0.5 & 0.896 & 1.250 \\
2 & $2.041 \times 10^{-1}$ & 1.120 & 1.221 \\
3 & $6.663 \times 10^{-2}$ & 1.361 & 1.387 \\
4 & $1.709 \times 10^{-2}$ & 1.615 & 1.615 \\
5 & $3.400 \times 10^{-3}$ & 1.879 & 1.870 \\
6 & $5.196 \times 10^{-4}$ & 2.150 & 2.139 \\
7 & $6.050 \times 10^{-5}$ & 2.430 & 2.417 \\
8 & $5.336 \times 10^{-6}$ & 2.708 & 2.701 \\
9 & $3.549 \times 10^{-7}$ & 2.996 & 2.988 \\
\hline
\end{tabular}

Table 5: Numerical estimation of the factor $A$

\begin{tabular}{crrll}
\hline$\nu$ & $\Delta$ & $C$ & $\gamma$ & $A$ \\
\hline 0.0 & 1.0 & 1.66925 & 0.0833 & 0.841 \\
0.2 & 0.8090 & 1.61803 & 0.1 & 0.816 \\
0.4 & 0.3090 & 1.49207 & 0.1722 & 0.747 \\
0.6 & -0.3090 & 1.33168 & 0.3833 & 0.68 \\
0.8 & -0.8090 & 1.16287 & 1.15 & 0.9 \\
\hline
\end{tabular}

Table 6: Comparison with exact values for $P(n)$ at $\Delta=1$

\begin{tabular}{cccc}
\hline$n$ & $P(n)$ & $\ln (P(n) / P(n+1))$ & Asymptotics (9) \\
\hline 1 & 0.5 & 1.58685 & 1.59489 \\
2 & $1.02284 \times 10^{-1}$ & 2.59643 & 2.59566 \\
3 & $7.62415 \times 10^{-3}$ & 3.60989 & 3.61060 \\
4 & $2.06270 \times 10^{-4}$ & 4.63019 & 4.62998 \\
5 & $2.01172 \times 10^{-6}$ & 5.65115 & 5.65133 \\
6 & $7.06812 \times 10^{-9}$ & & \\
\hline
\end{tabular}


conjecture with the factors given in Table 5 . We see that our first-principle data are well-fitted by our general formulas (7) and (8) for various values of $\nu$. However, as noted above, for some cases, there appear deviations.

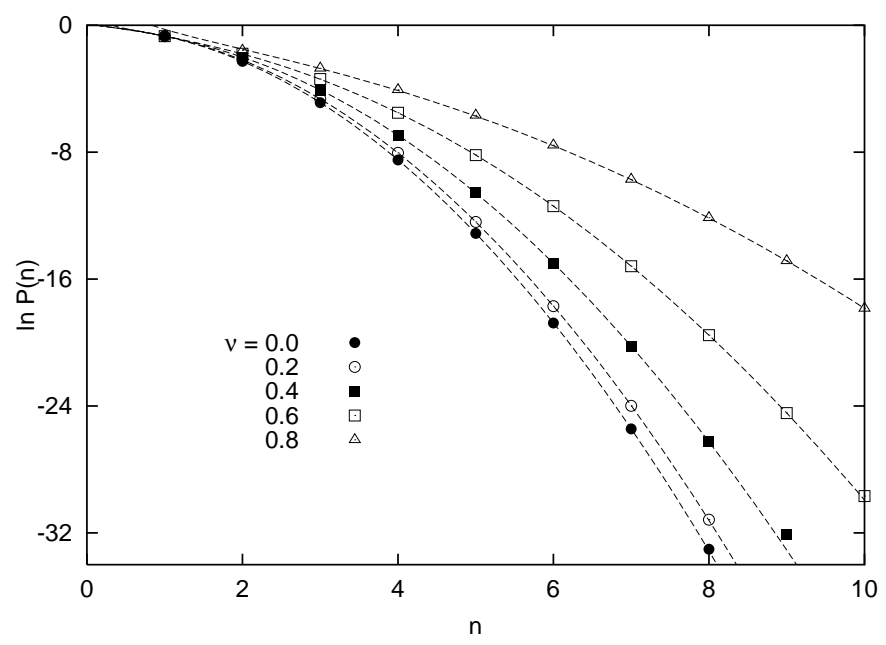

Figure 1: EFP is plotted for various valued of distances $n$ and anisotropies $\nu$. We have employed the DMRG method. The dotted lines are our conjecture (6) with the formulas (7) and (8).

Let us turn to addressing details of the DMRG method [13, 14, 15. DMRG is a sort of computer-aided real-space decimation, where the number of states for a renormalized block is retained within a bound tractable in computers. In this way, through the successive applications of DMRG, we are able to access very large system sizes. Hence, this method is suitable for surveying the long-distance asymptotic behavior of EFP. As a matter of fact, efficiency of the method was demonstrated in the preceding works [5, 8]: This success was rather unexpected, because in general, DMRG is not very efficient for such systems exhibiting criticality. In particular, long-distance asymptotic behavior of two-point correlation function is deteriorated rather severely. Those pathologies would be due to the truncation of bases through numerical renormalizations. However, as far as EFP is concerned, DMRG is proven to be free from such difficulties. Below, we will overview technical points of our numerical simulation. Our algorithm is standard [13], 14], and we refer readers to a recent proceeding 15] for full account of methodological details. We have employed the so-called infinite algorithm, which is adequate to investigate bulk properties at the ground state. (For the purpose of studying finite-size scaling behavior, the finite algorithm would be more suitable.) We have remained, at most, $m=300$ bases for a renormalized block. The density matrix eigenvalue $\left\{w_{\alpha}\right\}$ of remaining bases indicates the statistical weight of each remained state. We found $w_{\alpha}>5 \cdot 10^{-12}$ : That is, we have remained almost all relevant states with appreciable statistical weight $w_{\alpha}>5 \cdot 10^{-12}$, which may indicate error of the present simulation. We have repeated 300 renormalizations, and hence, total system size extends to $L=600$. The DMRG data alternates in turn through renormalizations: Note that the number of spins consisting a renormalized block increases by one after another through renormalizations. The problem is that the Hilbert-space structures are incompatible with respect to those cases whether the block contains even or odd spins. (Haldane system $(S=1)$ is not affected by this difficulty.) Therefore, we have taken arithmetic mean over those two cases.

The DMRG method is not efficient in the close vicinity of the ferromagnetic isotropic point $\nu=1$. Because in the vicinity of that point, the spin-wave velocity tends to vanish, and there appear numerous nearly-degenerated low-lying levels. Those nearlydegenerated levels are very hard to resolve in the process of numerical diagonalization. 
The diagonalization is a significant part in the DMRG procedure, and hence, DMRG becomes hardly applicable. In order to compensate this drawback, we have employed another numerical method, that is, the Monte-Carlo integration. We will explain it in the following.

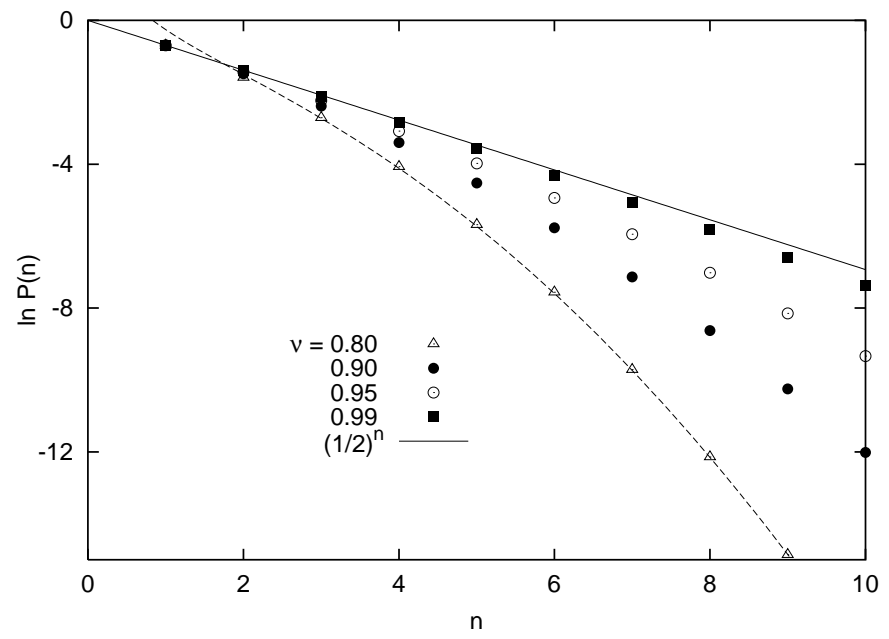

Figure 2: EFP is plotted for various valued of distances $n$ and anisotropies $\nu$. We have employed the Monte-Carlo integration method for Eq. (3). The dotted line is Eq. (6) for $\nu=0.8$.

In Fig. 2, we have presented numerical data with Monte-Carlo integration for the multiple integral formula (3) of Jimbo and Miwa. The technical details will be explained afterwards. From the plot, we see that the decay of EFP is gradually modified as for $\nu \rightarrow 1$, and surprisingly enough, the decay approaches the simple exponential formula of $(1 / 2)^{n}$ eventually. This feature is precisely in accord with the aforementioned finding that DMRG data deviate from the Gaussian formula as for $\nu \rightarrow 1$. Therefore, we see that at least for short-distance asymptotic form of EFP is governed by the simple exponential decay $0.5^{n}$ as $\nu \rightarrow 1$. As a matter of fact, right at $\nu=1$, one may easily verify the pure exponential decay $P(n)$ from the Jimbo-Miwa formula.

The crossover from Gaussian to exponential decay suggests that the quantum fluctuations are suppressed as $\nu \rightarrow 1$. As as matter of fact, field theoretical consideration [12] reveled that EFP measures the probability of formations of ferromagnetic islands with size $n$ surrounded by antiferromagnetic background from the viewpoint of Euclidean spacetime. Therefore, our result suggests that this Euclidean space-time picture is deteriorated for $\nu \rightarrow 1$, because the spin-wave velocity tends to vanish, and thus, the size of the islands grows along the imaginary-time direction abruptly.

In the following, we explain technical details of the Monte-Carlo integration. We have used the subroutine package described in the textbook 16]. As a random number generator, we have employed "Mersenne Twistor", MT19937 in Ref. [17]. For each plot, we have performed eight-billion Monte-Carlo steps. This main Monte-Carlo procedure is preceded by preliminary five million Monte-Carlo steps which are aimed to improve the efficiency of Monte-Carlo sampling by surveying the integrand in the multi-dimensional space. Irrespective of $n$ and $\nu$, we found that the statistical errors are of the order of $10^{-8}$.

To summarize, we have advocated compact explicit formulas (7) and (8) for the longdistance asymptotic form behavior of EFP. The formulas reproduce the presently-available exact results obtained at special solvable points of $\Delta=0$ and $1 / 2$, and therefore, we expect that our formulas are valid for over all the critical regime. We have performed extensive simulations of DMRG and Monte-Carlo integration. As a result, we found that 
our general formulas are indeed correct over the critical regime; see Tables and Fig. 1. In other words, EFP decays obeying the Gaussian formula (6) in the domain of criticality, and the decay rate becomes slower in the ferromagnetic side. This tendency is understood by a physical picture that EFP detects a formation of condensed particles (ferromagnetic string). Apparently, for ferromagnetic side, the condensation becomes promoted, and EFP gets enhanced, albeit the asymptotic form is still maintained to be Gaussian. However, as for the extreme limit to the ferromagnetic isotropic point, the Gaussian decay is smeared out by an exponential form at least for short distances. This fact reflects the suppression of quantum fluctuations in the ferromagnetic side. In other words, this crossover may be regarded as the precursor of the onset of true (long-range order) phase-separation for $\Delta<-1$, where EFP should not decay, but may saturate to a certain finite resident value. Finally we like to remark that the rate of Gaussian decay $C$, Eq.(7), can be evaluated analytically for some other rational values of $\nu$. For example, we have found

$$
\begin{aligned}
& \left.C\right|_{\nu=1 / 4}=2^{\frac{1}{4}} \mathrm{e}^{\frac{\mathrm{G}}{\pi}}, \quad(\mathrm{G}: \text { Catalan number }), \\
& \left.C\right|_{\nu=1 / 6}=2 \sqrt{\frac{2}{3}},\left.\quad C\right|_{\nu=2 / 3}=\frac{16(\sqrt{2}-1)}{3 \sqrt{3}},
\end{aligned}
$$

by use of the integral representations for the logarithm of (multiple) Gamma functions. These facts may indicate a possibility to prove our analytic formulas (6) rigorously for these values of $\nu$ just in the similar way as $\nu=1 / 2$ and $1 / 3$.

Acknowledgment We thank A. Abanov, H.Boos, F.Essler, F.Smirnov, M.Takahashi and A. Zamolodchikov for useful discussions. V. Korepin was supported by by NSF Grant PHY-9988566, S. Lukyanov was supported in part by DOE Grant DE-FG02-96ER40959, Y. Nishiyama was supported by Grant-in-Aid for Young Scientists No. 13740240, M. Shiroishi was supported by Grant-in-Aid for Young Scientists No. 14740228.

\section{References}

[1] M. Takahashi: J. Phys. C: Solid State Phys. 10 (1977) 1289.

[2] M. Jimbo and T. Miwa : "Algebraic analysis of solvable lattice models", AMS, 1995.

[3] M. Jimbo and T. Miwa : J. Phys. A 29 (1996) 2923.

[4] N. Kitanine, J. M. Maillet, V. Terras : Nucl. Phys. B 567, (2000) 554.

[5] M. Shiroishi, M. Takahashi, Y. Nishiyama : J. Phys. Soc. Jpn 70 (2001) 3535.

[6] N. M. Bogoliubov, A. G. Izergin, V. E.Korepin : "Quantum Inverse Scattering Method and Correlation Functions", Cambridge University Press, 1993.

[7] H. E. Boos and V. E. Korepin, : J. Phys. A: Math. Gen. 34 (2001) 5311.

[8] H. E. Boos, V. E. Korepin, Y. Nishiyama and M. Shiroishi: J. Phys. A: Math. Gen. 35 (2002) 4443.

[9] H. E. Boos, V. E. Korepin and F. A. Smirnov, hep-th/0209246.

[10] A. V. Razumov and Yu. G. Stroganov : J. Phys. A: Math. Gen. 34 (2001) 3185, ibid. 5335 .

[11] N. Kitanine, J. M. Maillet, N. A. Slavnov and V. Terras : J. Phys. A: Math. Gen. 35 (2002) L385.

[12] A. G. Abanov and V. E. Korepin : cond-mat/0206353.

[13] S. R. White : Phys. Rev. Lett. 69, 2863 (1992).

[14] S. R. White : Phys. Rev. B 48, 10345 (1993). 
[15] I. Peschel, X. Wang, M. Kaulke and K. Hallberg ed. : "Density-Matrix Renormalization - A New Numerical Method in Physics"", (Springer-Verlag, Berlin/Heidelberg 1999).

[16] W. H. Press, S. A. Teukolsky, W. T. Vetterling, and B. P. Flannery, Numerical recipes in FORTRAN (Cambridge university press, 1992).

[17] M. Matsumoto and T. Nishimura : ACM Trans. on Modeling and Computer Simulations, 8, 3 (1998). 